\title{
Four Taeniasis saginata Cases Diagnosed at a University Hospital in Korea
}

\author{
Eun Jeong Won ${ }^{1, *, \dagger}$, Ju Hyeon Shin², Yu Jeong Lee', Moon-Ju Kim¹, Seung Ji Kang ${ }^{3}$, Sook In Jung ${ }^{3}$, \\ Soo Hyun Kim², Jong Hee Shin², Jong-Yil Chai ${ }^{4}$, Sung-Shik Shin ${ }^{5, *,+}$ \\ ${ }^{1}$ Department of Parasitology and Tropical Medicine, Chonnam National University Medical School, Gwangju 61469, Korea; ${ }^{2}$ Department of \\ Laboratory Medicine, Chonnam National University Hospital, Gwangju 61469, Korea; ${ }^{3}$ Department of Infectious Diseases, Chonnam National \\ University Medical School, Gwangju 61469, Korea; ${ }^{4}$ Korea Association of Health Promotion, Seoul 07549, and Department of Parasitology and \\ Tropical Medicine, Seoul National University College of Medicine, Seoul 03080, Korea; ${ }^{5}$ Department of Parasitology, College of Veterinary Medicine, \\ Chonnam National University, Gwangju 61186, Korea
}

\begin{abstract}
In recent years, the taeniasis has been rarely reported in the Republic of Korea (Korea). But in this study, we intend to report 4 taeniasis cases caused by Taenia saginata during a 5-month period (February to June 2018) at a unversity hospital in Gwangju, Korea. Worm samples (proglottids) discharged from all cases were identified by phenotypic and molecular diagnostics. Mitochondrial cytochrome c oxidase subunit I sequences showed $99.4-99.9 \%$ identity with $T$. saginata but, differed by $4 \%$ from $T$. asiatica and by $7 \%$ from $T$. multiceps, respectively. We found that tapeworms in 2 cases (Cases 2 and 3) yielded exactly the same sequences between them, which differed from those in Cases 1 and 4 , suggesting intra-species variation in tapeworms. These taeniasis cases by $T$. saginata infection in this study, which occurred within a limited time period and region, suggest the possibility of a mini-outbreak. This study highlights the need for further epidemiological investigation of potentially overlooked cases of $T$. saginata infection in Korea.
\end{abstract}

Key words: Taenia saginata, intra-species variation, Korea

Taeniasis is the intestinal tapeworm infection caused by the 3 Taenia species, i.e., T. solium, T. saginata and T. asiatica [1]. This cestode disease occurs worldwide, even in developed countries; however, its epidemiology differs significantly among geographical areas [2-4]. The distribution of human taeniasis is related to cultural practices, including the consumption of undercooked meat or organs of intermediate hosts infected with viable metacestodes [3]. A nationwide survey in Korea found that the positivity rate for taeniid eggs had decreased from $1.85 \%$ in 1971 to $0.04 \%$ in 2013 [5,6]. Jeon et al. [7] reported that $T$. saginata accounted for $20.6 \%$ of Taenia tapeworms collected between 1935 and 2005 in Korea based on morphological and genetic analyses. Seven cases were diagnosed sporadically from 2006 to 2011 [1,8] and 4 additional cases were reported with molecular data at a referral laboratory

- Received 12 January 2019, revised 12 May 2019, accepted 23 May 2019.

*Corresponding authors (parasite.woni@jnu.ac.kr; sungshik@jnu.ac.kr)

†These authors equally contributed in this work.

(c) 2019, Korean Society for Parasitology and Tropical Medicine

This is an Open Access article distributed under the terms of the Creative Commons Attribution Non-Commercial License (http://creativecommons.org/licenses/by-nc/4.0) which permits unrestricted non-commercial use, distribution, and reproduction in any medium, provided the original work is properly cited. in 2013 [9]. Subsequently, there were no further reports of $T$. saginata infection, suggesting a near-virtual absence of $T$. saginata in Korea. Here, we summarize 4 cases of taeniasis caused by $T$. saginata during a 5-month period from February to June 2018 encountered at Chonnam National University Hospital, Gwangju, Korea.

As summarized in Table 1, all of 4 patients lived in Gwangju and suffered from intermittent passage of proglottids in the stool for several months. First, a 49-year-old woman visited to the hospital in February 2018 (Fig. 1A). She had had postprandial abdominal discomfort for the previous year and noticed proglottids in her stool for 1 month. She enjoyed eating raw beef and fish, but not raw pork or liver. Routine laboratory tests showed moderate eosinophilia $(9.6 \% ; 1,000 / \mu \mathrm{l})$ and mild pyuria (white blood cells 1+). Ova of Taenia species were found on stool examination using the formalin-ether concentration method (Fig. 1B) [10]. Some proglottid segments expelled after praziquantel treatment were submitted to the Clinical Parasitology Laboratory. The segments were pressed between 2 microscope slides and examined macroscopically without staining, but the morphological characteristics such as 

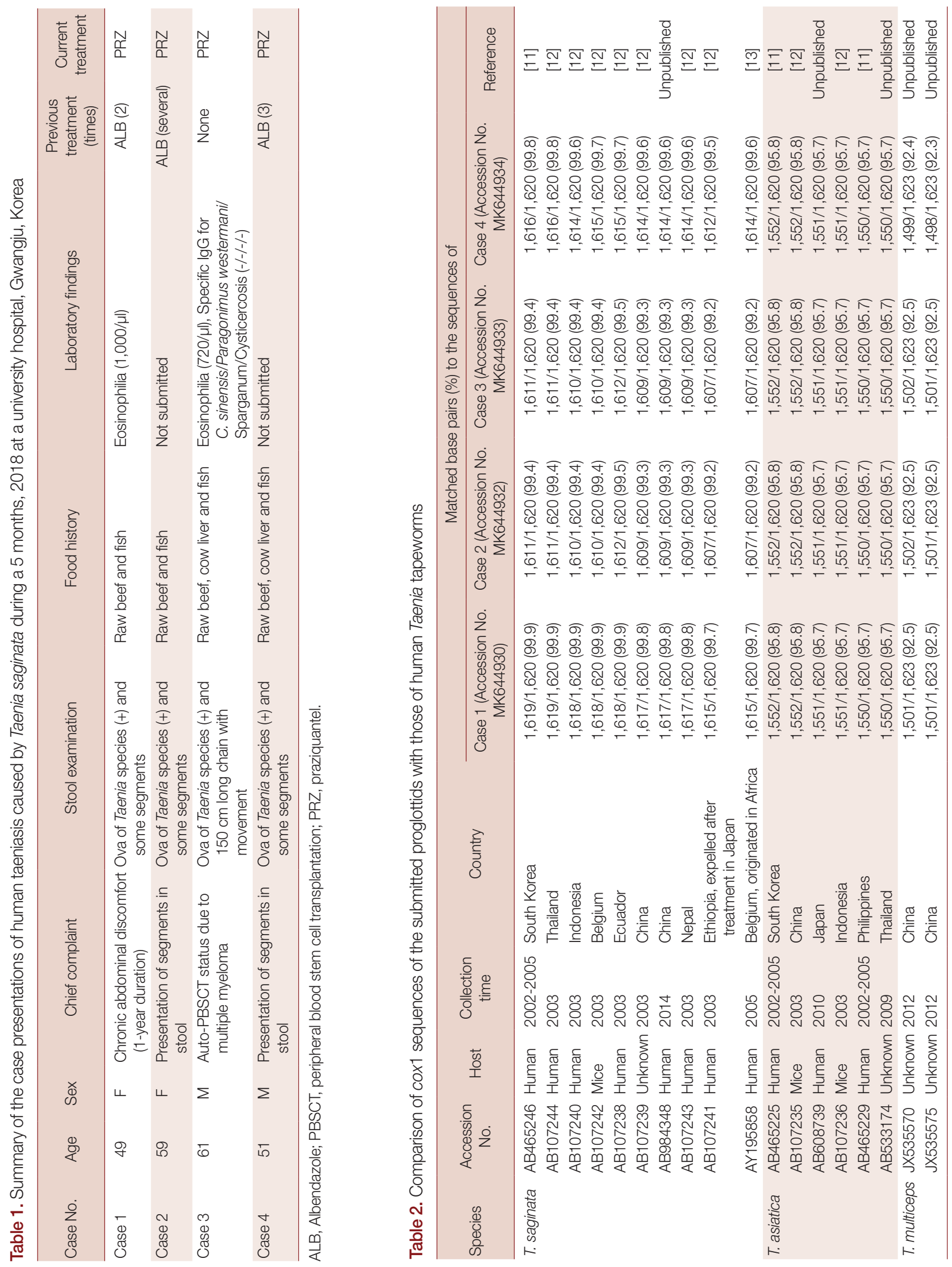

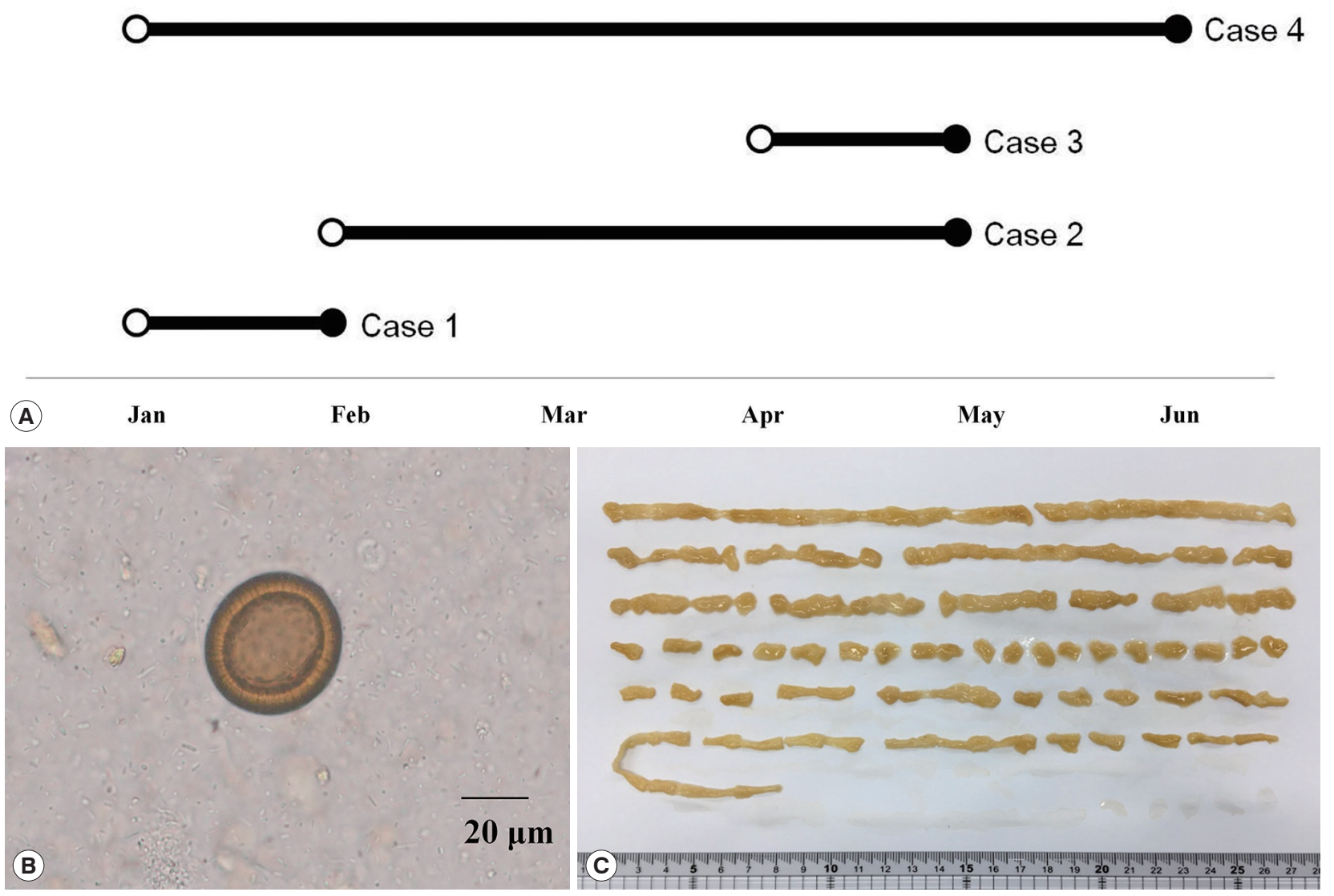

Fig. 1. Summary on 4 taeniasis saginata cases. (A) Four taeniasis cases occurred in the first half of 2018. An open circle indicates first proglottid passage; a closed circle indicates hospital visit. (B) An egg of Taenia detected from a case. (C) Segmented strobila $150 \mathrm{~cm}$ long, from Case 3.

uterine segments were not clear. A 59-year-old woman (Case 2) visited to our hospital in May 2018. She had noticed the intermittent passage of proglottids in her stool for 3 months, despite several albendazole treatments. She also enjoyed consuming raw beef and fish, but not raw pork or liver. No laboratory tests were requested except for the stool examination. Ova of Taenia species were found on the stool examination using the formalin-ether concentration method. We found more than 15 uterine segments filled with eggs in a proglottid pressed between 2 slides. A 61-year-old man (Case 3) also visited to our hospital in May 2018. He had been diagnosed with multiple myeloma, IgG, lambda type in October 2017 and had undergone chemotherapy followed by autologous peripheral blood stem cell transplantation in February 2018. He noticed the passage of proglottids in his stool 3 times during 1 month. He had consumed raw beef, raw cow liver, and raw fish, but not raw pork. Laboratory tests showed moderate eo-

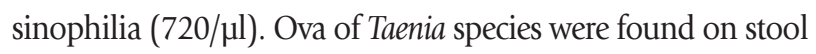

examination using the formalin-ether concentration method. An approximately 150-cm-long segment chain was collected after treatment with praziquantel (600 mg, 3 times a day) (Fig. 1C). We observed more that than 15 uterine segments filled with eggs in the expelled proglottids. A 51-year-old man (Case 4) visited to our hospital in June 2018 complaining of intermittent passage of proglottids in his stool for 5 months. He enjoyed raw beef, raw cow liver, or raw fish, but not raw pork. Any laboratory test was not requested except for the stool examination. A stool examination detected ova of Taenia species using the formalin-ether concentration method. A piece of proglottid was submitted, but was insufficient for morphological differentiation. All 4 patients were treated with praziquantel $(10 \mathrm{mg} / \mathrm{kg})$, and no ova were detected at follow-up.

To identify the tapeworm species, a partial sequence of the mitochondrial cytochrome $c$ oxidase subunit 1 ( cox1) gene was amplified from the submitted tapeworm segments. Genomic DNA was extracted from each ethanol-preserved tape- 


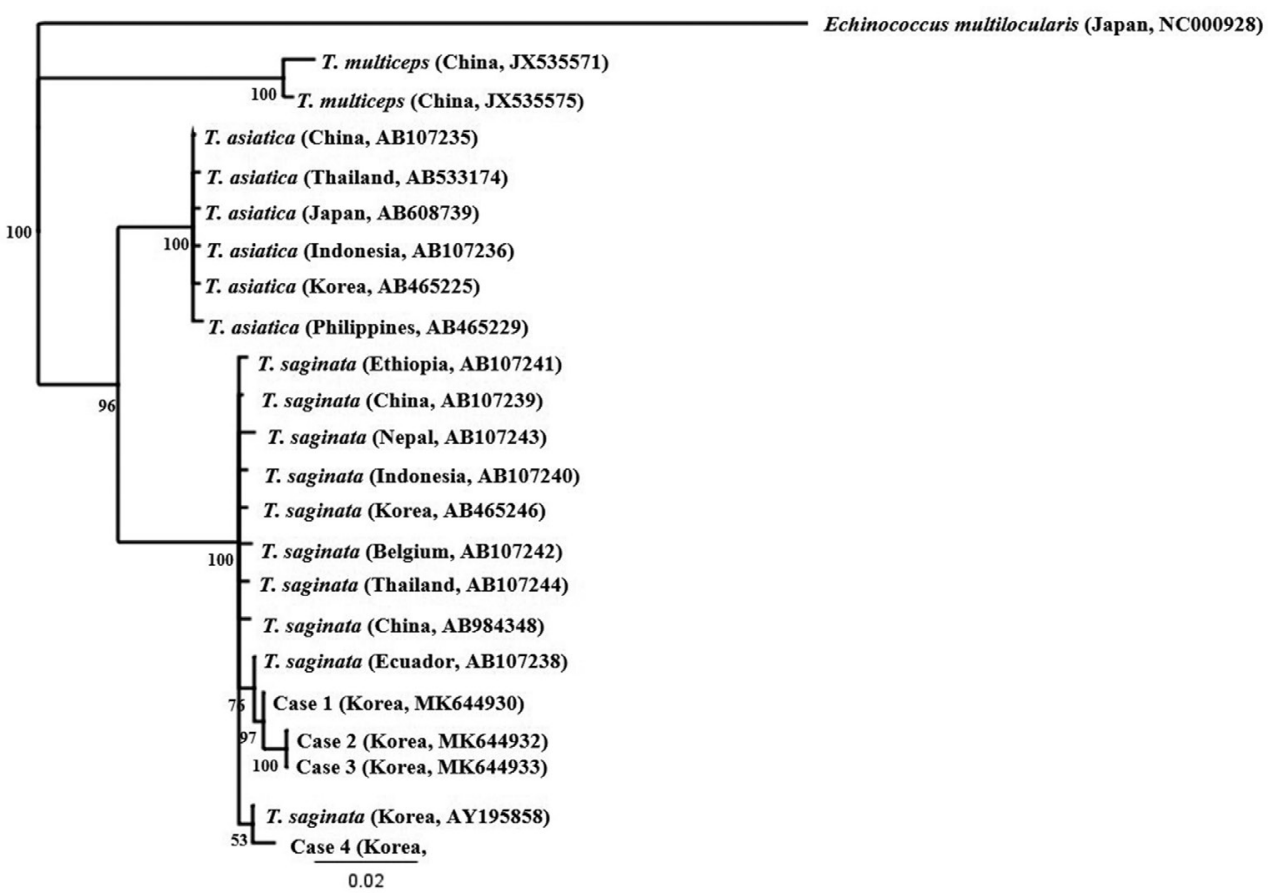

Fig. 2. A phylogenetic tree drawn based on cox1 sequences of taeniid tapeworms collected in this study and retrieved from GenBank. Accession numbers are indicated. Scale bar indicates a sequence distance of 0.02 nucleotide substitutions per site.

worm segment using the QIAamp DNA Mini Kit (Qiagen Hilden, Germany), according to the manufacturer's protocol, and used as a template for polymerase chain reaction (PCR) with the primers Tsag_cox1/F (5'-GAGGAAATTGTGAAGTTACTGCTA-3') and Tsag_cox1/R (5'-ATGATGCAAAAGGCAAATAAACCT- $3^{\prime}$ ) [11]. To amplify the cox1 gene, PCR was performed in $15 \mu$ reaction mixtures containing $1 \mu$ lemplate, $200 \mu \mathrm{M}$ of each dNTP, $0.2 \mu \mathrm{M}$ of each primer, $0.3 \mathrm{U}$ of Ex Taq polymerase (TaKaRa, Shiga, Japan) and manufacturer-supplied reaction buffer. The PCR cycling conditions consisted of 35 cycles of denaturation at $94^{\circ} \mathrm{C}$ for $30 \mathrm{sec}$, annealing at $66^{\circ} \mathrm{C}$ for $30 \mathrm{sec}$, and extension at $72^{\circ} \mathrm{C}$ for $90 \mathrm{sec}$. The PCR products were sent to Macrogen (Seoul, Korea) for direct sequencing using the PCR primers listed above. The chromatograms of both sequences were trimmed manually and assembled using SeqMan software (DNASTAR, Madison, Wisconsin, USA). Using BLAST searches, the assembled sequences, ranging in size from 1,762 to 1,791 bp, were compared Platyhelminths sequences deposited in the GenBank database. The cox1 PCR products amplified from expelled segments showed 99.4-99.9\% identity with T. saginata (GenBank no. AB465246; Korea), but differed by $4 \%$ from T. asiatica ( $95.8 \%$ identity with the sequence of AB465225) and by 7\% from T. multiceps (92.5\% identity with the sequence of JX535575), respectively (Table 2). To understand phylogenetic relationships, the assembled sequences of the 4 cases were compared with those of T. saginata from Korea (AB465246), China (AB107239 and AB984348), Japan (AB107241), Indonesia (AB107240), Belgium (AB107242 and AY195858), Ecuador (AB107238), Thailand (AB107244), and Nepal (AB107243); with those of T. asiatica from Korea (AB465225), China (AB107235), Japan (AB608739), Indonesia (AB107236), Philippines (AB465229), and Thailand (AB533174); with those of Taenia multiceps from China (JX535570 and JX535575), respectively [11-13]. The phylogenetic relationships among the Taenia genotypes were inferred using neighbor-joining (NJ) analysis with Geneious software (11.1.3) based on Tamura-Nei genetic distance model. Bootstrap analysis was performed with 3,000 replications. The mitochondrial cox 1 gene sequence of Echinococcus multilocularis (NC000928) was used as an outgroup for phylogenetic analysis of cox 1 dataset. Our 4 sequences clustered with the T. saginata cox 1 genes, distinct from those of both other Taenia species (Fig. 2). The clustering diagram of T. saginata cox1 gene indicated no significant genetic differentiation among different countries, although one of the 4 sequences appeared to be separated slightly. 
Human taeniasis is usually diagnosed by observing ova in a patient's stool or examining gravid proglottids. All of the patients in this study visited the hospital because of passage of tapeworm proglottids in the feces. Morphological identification is essential, but it requires expertise and experience to identify Taenia species accurately. Moreover, observation of the uterine structure can be ambiguous when the specimen is not fresh [9]. T. asiatica can be misidentified as T. saginata because of their morphological similarity without molecular analysis $[7,9]$. We correctly differentiated T. saginata from T. asiatica by direct sequencing of the cox 1 gene. Furthermore, we found that tapeworms in Cases 2 and 3 had exactly the same sequences, but differed from those in Cases 1 and 4, suggesting intra-species variation in tapeworms. Little is known about genotype of T. saginata obtained from Korean patients. Anantaphruti et al. [14] found 14 haplotypes among $73 \mathrm{~T}$. saginata isolates in Thailand by partial cox1 sequencing (924 bp): haplotype A was the major one (52.1\%), followed by haplotype B (21.9\%). Our Cases 1 and 4 seem to be haplotype A, while Cases 2 and 3 were new haplotype (A12G and C194T, compared to haplotype B). Jeon et al. [13] performed partial nucleotide sequencing of mitochondrial cox1 and ITS2 of Taenia specimens from Korean collections obtained over 70 years and found that $T$. asaitica accounted for 3-quarters of all Taenia infections, and that there was no significant intra-species variation in tapeworms. Recently, human infections caused by hybrid infection with T. saginata and T. asiatica tapeworms in Laos were identified by sequencing the DNA polymerase delta region [4]. Although we could not perform this, further study may be necessary to understand genetic variation using both mitochondrial and nuclear DNA.

Human taeniasis has virtually disappeared during the past 2 decades in Korea, although a few sporadic cases may be hidden, or the exact prevalence may be underestimated. It is noteworthy that our 4 cases occurred within a limited area and a short time period. This suggests the possibility that cattle harboring T. saginata metacestodes may be present in Korea. Little has been reported about the current epidemiology on Cysticercus bovis in cattle, although the positive rates of 5.1-37.6\% were reported from 1924 to 1943 [15-17]. Very recently, the Health and Environment Research Institute of Gwangju investigated the seroprevalence of cysticercosis in 213 serum samples collected from 2 slaughterhouses in Gwangju from April to July 2017 using an enzyme-linked immunosorbent assay (ELISA). A single sample obtained from a beef cattle farm with more than 100 cows in Jeollanam-do was positive for cysticercosis. ELISA is more sensitive than classical meat inspection techniques for identifying cattle infected with cysticerci [18]. Although we could not track the exact origin of the raw beef in this study, further inspection may be helpful to clarify whether the lifecycle of T. saginata persists in nearby Jeollanam-do. Infection caused by imported beef or the past infection are also possible, other than domestic mini-outbreak. Nevertheless, clinician should be aware of this overlooked parasitic infection considering the traditional habit of ingesting undercooked beef in Korea. Our findings also suggest the need for further epidemiological evaluation of T. saginata infection in Korea.

\section{ACKNOWLEDGMENT}

This study was supported by a grant (CRI17009-1) of Chonnam National University Hospital Biomedical Research Institute.

\section{CONFLICT OF INTEREST}

We have no conflict of interest related to this work.

\section{REFERENCES}

1. Chai JY. Human taeniasis in the Republic of Korea: hidden or gone? Korean J Parasitol 2013; 51: 9-17.

2. Okello AL, Thomas LF. Human taeniasis: current insights into prevention and management strategies in endemic countries. Risk Manag Healthc Policy 2017; 10: 107-116.

3. Dorny P, Praet N. Taenia saginata in Europe. Vet Parasitol 2007; 149: 22-24.

4. Sato MO, Sato M, Yanagida T, Waikagul J, Pongvongsa T, Sako Y, Sanguankiat S, Yoonuan T, Kounnavang S, Kawai S, Ito A, Okamoto M, Moji K. Taenia solium, Taenia saginata, Taenia asiatica, their hybrids and other helminthic infections occurring in a neglected tropical diseases' highly endemic area in Lao PDR. PLoS Negl Trop Dis 2018; 12: e0006260.

5. Ministry of Health and Social Affairs and Korean Association for Parasite Eradication. Prevalence of Intestinal Parasitic Infections in Korea-the 1st report. Seoul, Korea. 1971, pp 1-26 (in Korean).

6. Korea Centers for Disease Control and Prevention. National Survey of Intestinal Parasitic Infections in Korea, 8th Report 2013. KCDC 2014; 1: 89-94 (in Korean).

7. Jeon HK, Kim KH, Chai JY, Yang HJ, Rim HJ, Eom KS. Sympatric distribution of three human Taenia tapeworms collected between 1935 and 2005 in Korea. Korean J Parasitol 2008; 46: 235-241. 
8. Choi SC, Lee SY, Song HO, Ryu JS, Ahn MH. Parasitic infections based on 320 clinical samples submitted to Hanyang University, Korea (2004-2011). Korean J Parasitol 2014; 52: 215-220.

9. Cho J, Jung BK, Lim H, Kim MJ, Yooyen T, Lee D, Eom KS, Shin EH, Chai JY. Four cases of Taenia saginata infection with an analysis of COX1 gene. Korean J Parasitol 2014; 52: 79-83.

10. Won EJ, Kim J, Ryang DW. Evaluation of modified formalinether concentration method using para tube in clinical settings. Ann Lab Med 2015; 35: 445-448.

11. Okamoto M, Nakao M, Blair D, Anantaphruti MT, Waikagul J, Ito A. Evidence of hybridization between Taenia saginata and Taenia asiatica. Parasitol Int 2010; 59: 70-74.

12. Yamasaki H, Allan JC, Sato MO, Nakao M, Sako Y, Nakaya K, Qiu D, Mamuti W, Craig PS, Ito A. DNA differential diagnosis of taeniasis and cysticercosis by multiplex PCR. J Clin Microbiol 2004; 42: 548-553.

13. Jeon HK, Eom KS. Taenia asiatica and Taenia saginata: genetic divergence estimated from their mitochondrial genomes. Exp Par- asitol 2006; 113: 58-61.

14. Anantaphruti M, Thaenkham U, Kusolsuk T, Maipanich W, Saguankiat S, Pubampen S, Phuphisut O. Genetic variation and population genetics of Taenia saginata in north and northeast Thailand in relation to Taenia asiatica. J Parasitol Res 2013; 2013: 310605.

15. Nakanishi S. Statistical observation of Cysticercus inermis in native Korean calves. Nippon Jui Gakkai Zasshi 1926; 5: 147-150 (in Japanese).

16. Isshiki O. Intestinal helminth infections of domestic animals in Cheju. Chosen GakuHo 1960; 16: 1-13 (in Japanese).

17. Eom KS, Rim HJ. Epidemiological understanding of Taenia tapeworm infections with special reference to Taenia asiatica in Korea. Korean J Parasitol 2001; 39: 267-283.

18. Dorny P, Vercammen F, Brandt J, Vansteenkiste W, Berkvens D, Geerts S. Sero-epidemiological study of Taenia saginata cysticercosis in Belgian cattle. Vet Parasitol 2000; 88: 43-49. 